\title{
The initiation of metabolic inflammation in childhood obesity
}

\author{
Kanakadurga Singer' and Carey N. Lumeng ${ }^{1,2}$ \\ 'Department of Pediatrics and Communicable Diseases and 'Molecular and Integrative Physiology, University of Michigan Medical School, Ann Arbor, Michigan, USA
}

\begin{abstract}
An understanding of the events that initiate metabolic inflammation (metainflammation) can support the identification of targets for preventing metabolic disease and its negative effects on health. There is ample evidence demonstrating that the initiating events in obesity-induced inflammation start early in childhood. This has significant implications on our understanding of how early life events in childhood influence adult disease. In this Review we frame the initiating events of metainflammation in the context of child development and discuss what this reveals about the mechanisms by which this unique form of chronic inflammation is initiated and sustained into adulthood.
\end{abstract}

\section{Introduction}

The prevalence of obesity and metabolic disease continues to increase from year to year in the US adult population (1) and generates an immense burden on quality of life and healthcare expenditures (2). More ominously, the rate of childhood obesity has increased in parallel with adult obesity, resulting in pediatric patients presenting with diseases traditionally associated with adulthood such as dyslipidemia, nonalcoholic fatty liver disease (NAFLD), and type 2 diabetes. Despite some reports to the contrary, the latest data show that the prevalence of childhood obesity between 1999 to 2014 has not declined and continues to increase in all age groups $(17.4 \%$ of children have a BMI greater than the 95th percentile for their age and sex) (3). More alarmingly, $10.5 \%$ of adolescents meet the pediatric modified criteria for metabolic syndrome (4), a prevalence that exceeds that of diseases such as asthma and food allergies in children.

The insidious effects of metabolic disease and the limited treatments to prevent the long-term morbidities of metabolic syndrome on cardiovascular disease (CVD) make obesity a threat to both child and adult health. Longitudinal studies demonstrate that the early onset of dyslipidemia and high blood pressure in children is associated with premature atherosclerosis in adults (5). Therefore, an understanding of the initiating events that occur in children that contribute to insulin resistance and CVD has the potential to positively influence how we treat and prevent metabolic disease and its morbidities in all ages. These problems are compounded by the fact that most pediatric caregivers are restricted in their abilities to manage metabolic disease and obesity due to limited resources for nutrition and exercise counseling, the need for multidisciplinary teams for intensive obesity treatment, and the lack of medical treatments available for pediatric obesity.

The evidence above suggests that obesity-associated metabolic dysfunction can be framed as a disease of childhood in which growth, development, the environment, and genetic pre-

Conflict of interest: The authors have declared that no conflict of interest exists. Reference information: J Clin Invest. 2017;127(1):65-73. doi:10.1172/JCI88882. disposition are intertwined. Such a framework provides a novel perspective on the potential mechanisms by which inflammation and metabolism interact over the lifespan and may change the face of metabolic disease treatment and prevention for decades to come. Many of the developmental windows that are sensitive to the patterning of metabolic physiology are also crucial in the patterning of the immune system and its responses. As such, in the setting of obesity, codependent interactions between metabolism and inflammation have been observed in the prenatal environment, infancy, early childhood, and adolescence. In this Review we highlight the evidence that metabolic inflammation (hereafter referred to as metainflammation) is initiated in childhood and discuss the mechanisms by which this is triggered in the context of child development.

\section{What is the nature of the inflammatory response to obesity?}

Inflammation is the coordinated biologic response to the disruption of normal cellular or systemic physiology. Disruptors can be external pathogens such as bacteria, intrinsic disturbances such as cell death or an oncologic process, or physiologic alterations in response to environmental signals such as fasting or overfeeding. Host inflammatory responses can be acute with the goal of eliminating the effects of a negative stimulus and restoring the system back to its state prior to the disruption (e.g., rapid control of local infection). Chronic inflammation can be seen in circumstances in which rapid clearance mechanisms fail or are incomplete or are generated by gradual or repeated alterations in normal physiology, such as obesity. The protective goal of restoration of homeostasis is retained with chronic inflammation and therefore involves many of the same patterned responses utilized in acute inflammatory settings, such as the production of chemokines, leukocyte activation, activation of innate pattern recognition receptors, and activation of adaptive immunity.

In chronic metabolic disease, inflammatory mediators are both dominant and antagonistic to the homeostatic system of controllers and sensors that regulate physiologic variables such as blood glucose $(6,7)$. Metabolic disease-associated inflamma- 


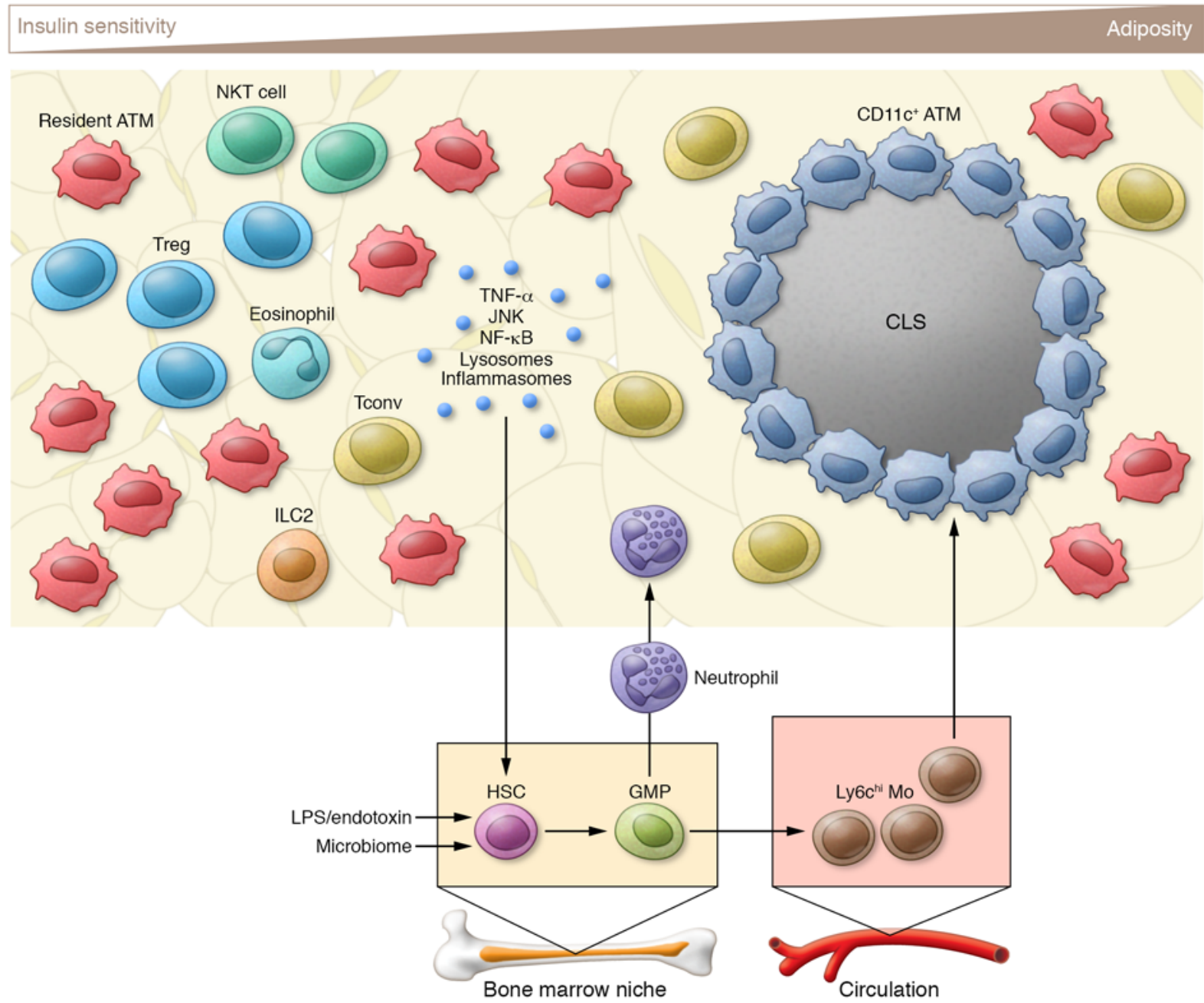

Figure 1. The changing inflammatory environment in adipose tissue with obesity. The progression from a lean to obese state leads to adipocyte expansion. The lean environment contains numerous leukocytes that play regulatory roles in maintaining low inflammatory tone in adipose tissue. These serve to buffer acute metabolic changes in adipose tissue nutrient storage and return the system to baseline. Chronic obesity leads to early events such as conventional T cell (Tconv) activation and neutrophil accumulation that enhance adipocyte damage and CLS formation. A high proinflammatory tone is triggered and maintained by signals from adipose tissue and the environment that drive HSC expansion and production of monocytes (Mo) and neutrophils from granulocyte/macrophage progenitors (GMPs). The net result is the sustained accumulation and activation of ATMs that potentiate continued adipose tissue dysfunction.

tion, or metainflammation, can be detected in numerous tissues involved in nutrient regulation. Since the early 1970s, adipose tissue has been recognized as a nexus between inflammation and metabolism with the demonstration that endotoxin amplifies the release of free fatty acids from visceral adipose tissue (VAT) (8). The identification of the chronic release of cytokines such as TNF- $\alpha$ from obese adipose tissue initiated a wave of studies that have unveiled a complex and integrated adipose tissue immune system that includes both innate and adaptive components (refs. 9, 10, and Figure 1). Within this network, numerous innate inflammatory signaling pathways such as the inflammasome, lysosomes, NF- $\mathrm{KB}$, and JNK are activated in adipose tissue and contribute to metabolic disruption (11-14). These acute inflammatory signals may not always antagonize metabolic adaptation to over-nutrition and in fact may be required for the appropriate response in adipose tissue. Proinflammatory cascades within adipocytes are required for adipogenesis and adipocyte hypertrophy with high-fat diet (HFD) challenge (15). During a short-term HFD challenge in mice, insulin resistance develops independent of inflammation and is characterized by NKT cellmediated promotion of alternative activation of resident adipose tissue macrophages (ATMs) $(16,17)$. Thus, inflammatory responses in this context likely represent an adaptive response that permits healthy adipose tissue expansion and efficient lipid storage. The nature of the inflammatory response in short-term HFD exposure may be tissue specific, as reactive gliosis and neuronal damage are observed in the hypothalamus in conjunction with proinflammatory signals (18).

With chronic obesity, leukocytes within adipose tissue generate a sustained proinflammatory tone that has negative effects on adipocyte insulin sensitivity and contributes to systemic insulin resistance (17). Adipose tissue leukocytes such as eosinophils and innate lymphoid cells interact with ATMs in the lean state to maintain homeostasis and counteract the proinflammatory signals (19). 


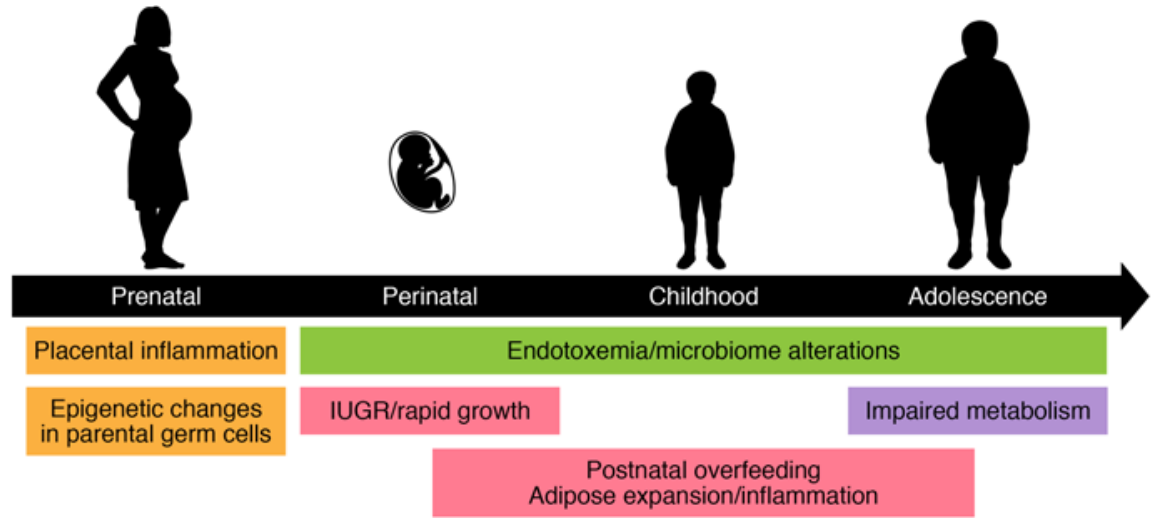

Figure 2. A developmental framework for the initiation of metainflammation. Throughout the lifecourse there are multiple initiating events that can lead to the expansion of adipose tissue and systemic chronic inflammation. These include programmed inheritance (orange), environmental factors (green), intrinsic growth rates of the child (red), and adipose tissue metabolic impairments (purple). increase in ATM infiltration and amplification of immune responses associated with hypomethylation in innate pattern recognition genes such as Tlr1 and Tlr2 (36).

The clinical evidence that obesity during pregnancy can lead to proinflammatory activation in the fetus is somewhat sparse (37). In obese mothers, placental inflammatory macrophages are increased and produce proinflammatory cytokines, although fetal blood leukocytes do not appear to be altered $(38,39)$. Maternal obesity and overweight status are also risk factors for increased serum inflammatory markers in preterm infants, but not in term newborns (40). After adjusting for childhood BMI, C-reactive protein (CRP) levels were increased in 12-yearold children born to mothers with higher In mice and humans, qualitative and quantitative changes in ATMs occur in obesity that are mediated by proliferation and leukocyte trafficking from the circulation $(20,21)$. Events that precede and may amplify ATM recruitment include the activation of conventional $\mathrm{CD} 4^{+} \mathrm{T}$ cells and the accumulation of adipose tissue neutrophils (22-24). Recruited ATMs prominently express the marker CD11c in mice and home to crown-like structures (CLSs), which form around dead or dying adipocytes and are a recognized sign of adipose tissue dysfunction in many contexts $(25,26)$. ATMs assume phenotypic changes characterized by lysosomal activation and the formation of a metabolically activated phenotype distinct from classical (M1) or alternative (M2) activation $(13,27)$. Adaptive immunity is also triggered by chronic inflammation via the function of ATMs and adipose tissue dendritic cells as antigen-presenting cells $(28,29)$. This promotes Th1 immunity in adipose tissue by stimulating conventional $\mathrm{T}$ cell activation at the expense of Tregs $(24,30)$.

\section{When does metainflammation begin?}

The increase in obesity prevalence at all ages suggests that the initiating events for metainflammation may occur in the earliest developmental stages. This concept is emphasized in the developmental origins of health and disease paradigm, which postulates that prenatal and perinatal factors influence adult diseases such as diabetes and obesity via epigenetic and physiologic programming (31). There is evidence to support the induction of metainflammation in all developmental stages, with the potential for life-long consequences on metabolic regulation as well as immune responses to non-metabolic stimuli (Figure 2). These include factors both intrinsic and extrinsic to the developing child that influence metabolic and inflammatory responses.

Prenatal period. The prenatal period can be influenced by multiple factors from both the maternal and paternal environment. Rodent studies suggest that maternal obesity enhances adipose tissue inflammation in the offspring independent of offspring adiposity (32-34). Paternal obesity leads to epigenetic alterations in the sperm in rodents and can potentiate hypothalamic inflammation in offspring (35). Similar epigenetic mechanisms have been observed in a multigenerational HFD feeding model in mice that lead to an pre-pregnancy BMIs, suggesting that maternal obesity may alter the immune system in the long term (41).

In terms of inflammation and disease risk, low birth weight (LBW) is a well-documented and strong risk factor for metabolic disease - a concept encompassed in the Barker hypothesis linking poor in utero growth to CVD and diabetes (42-44). In rodents, intrauterine growth restriction (IUGR) is associated with increased inflammatory markers in the circulation of the offspring (45-47). Proposed mechanisms for this effect include alterations in the circadian clock and downregulation of Pparg and Ppara with IUGR (48). Longitudinal studies such as the Bogalusa Heart Study also show that LBW induces chronic elevations in wbc counts and inflammatory biomarkers such as CRP in childhood $(49,50)$. Similar to LBW, high birth weight is associated with increased adult visceral adiposity and insulin resistance; however, the evidence that this occurs in association with elevated levels of inflammatory biomarkers such as CRP is not strongly supported $(51,52)$. These findings suggest that for some children the initiating events of metainflammation may occur in the postnatal setting and less so in the prenatal environment.

Perinatal/infancy period. The transition from the intrauterine to the extrauterine environment during the perinatal period is characterized by intense remodeling of both metabolic physiology and the immune system. These two processes intersect in places like the gut where the establishment of the intestinal microbiome after birth is coupled to the development of the immune system that is permissive towards colonization in early life (53). The immune system in infants is immature and over time transitions from one dependent primarily on innate immunity for host defense to one that is able to mount adaptive responses to pathogens over the first years of life (54). This early post-natal stages are also a critical window where tissue resident leukocytes such as Tregs take residence in adipose tissue (55). Events that disrupt or modify the developing immune system in this critical window may have long-term consequences for health and disease.

Preclinical studies using HFD to model perinatal nutrient excess demonstrate activation of JNK and IKB kinase subunit $\beta$ (IKK $\beta$ ) pathways in the liver and hypothalamus in association with insulin 
resistance and fatty liver in the offspring $(56,57)$. Rodent models of postnatal overnutrition and accelerated growth by manipulation of litter size show that a HFD may not be required to trigger inflammation and generate lifelong effects on metabolic dysfunction (5860). Postnatal overfeeding (PNOF) in rats is sufficient to induce hypothalamic inflammation and gliosis as early as postnatal day 14 and leads to a persistent increase in hypothalamic NF- $\kappa \mathrm{B}$ activity in adulthood $(61,62)$. PNOF can enhance microglial activation in response to HFD in adults, which suggests that the inflammatory set point in the brain is permanently modified with accelerated early life growth $(63,64)$. The mechanism of the persistent alterations in hypothalamic inflammation with PNOF may be different than HFD-induced hypothalamic inflammation in adult mice, which is reversible $(18,65)$. PNOF can also amplify adipose tissue inflammation in response to a HFD in adulthood and can also increase ATM infiltration and CLS formation $(66,67)$. Reprogramming of inflammatory responses by perinatal overnutrition does not seem to be limited to metainflammation, as PNOF can alter global responses to pathogen-associated molecular pattern molecules such as LPS and modulate pulmonary inflammation $(68,69)$.

While there are few clinical studies focused on the inflammatory effects of rapid infant growth, they demonstrate a consistent finding that rapid postnatal growth in the first 6 to 12 months of life is a strong risk factor for metabolic disease independent of birth weight (70-73). In fact, the loci that contribute genetic susceptibility to obesity play a dominant role in regulating weight and fat mass in the first three years of life but do not appear to affect birth weight (74). Weight gain in infancy is a risk factor for elevated CRP and great carotid intima-media thickness in non-diabetic children (75). Large cohort studies have identified surprising links between infant growth and inflammatory diseases such as type 1 diabetes (76) and asthma (77), suggesting that early rapid growth rates may have broad effects on inflammatory responses to environmental stimuli during childhood. Overall, the immediate postnatal period is clearly a sensitive window for the patterning of nutritional and inflammatory responses that is understudied in the context of metainflammation. Future work is needed in this area, as this time period may be amenable to interventions in the primary care setting, in which growth rates are closely monitored but few guidelines are set regarding how to manage infants with rapid growth.

Childhood/adolescence. While many preclinical studies have examined the effects of obesity on inflammation in prenatal and early postnatal periods, there are limited basic studies assessing the effects of childhood and adolescent obesity on life-long disease risk. Clinically, obese children are more likely to become obese adults (78), and excess BMI and high BMI trajectories in adolescents are associated with a future risk of adult diabetes (79). The observation that normal-weight adults who were obese only as children retain a four-fold increased risk for type 2 diabetes further suggests that the effects of childhood obesity can be long lasting, even if BMI is normalized by adulthood (80).

Evidence is strong that metainflammation is triggered by childhood obesity. Overweight and obese children as young as 3 years of age demonstrate elevations in CRP and absolute neutrophil counts $(81,82)$. Several studies have shown that elevated wbc counts in obese children track into adulthood and correlate with the number of metabolic syndrome components (83). Rapid weight gain during childhood predicts both future weight gain (84) and CRP levels (85). In children, these inflammatory biomarkers are strongly associated with adiposity (86), and in severely obese adolescents the risk of elevated CRP, impaired fasting glucose, and hypertension increase in concert with increased BMI $(87,88)$. Protective factors such as adiponectin have been shown to have a similar decrease with increasing BMI and increased CRP (88).

Beyond these changes in circulating biomarkers, tissuespecific inflammation in obese children parallels what is seen in obese adults. NAFLD is present in $59 \%$ of adolescents referred for bariatric surgery, with $10 \%$ of these showing evidence of nonalcoholic steatohepatitis (NASH) (89). Severity of NAFLD in this cohort correlated with wbc count, and liver samples from adolescents with NASH had increased expression of genes involved in macrophage chemotaxis. Obese adolescents with NAFLD have increased CLSs in their subcutaneous adipose tissue that correlates with liver fibrosis independent of BMI (90). Adipose tissue in obese adolescents, similar to adults, has ATM infiltration and evidence of inflammasome activation that correlates with decreased insulin sensitivity (91). Adipose tissue CLSs have been identified in children as young as six years old in association with elevated CRP (92). Overall, these findings demonstrate that adipose tissue metabolic stress and inflammation are common in obese children and adolescents and may set the stage for decades of dysfunctional adipose tissue.

\section{What are the initiators of metainflammation?}

Characterizing the events that initiate metainflammation in children may be key to identifying prevention strategies throughout the lifespan and identifying those individuals most at risk for metabolic disease morbidities. Inflammation is observed in a range of tissues involved in nutrient control. For example, proinflammatory macrophage signatures are observed in sites that include adipose tissue, gut (93), liver (94), pancreas (95), muscle (96), and CNS $(18,97)$. Animal models suggest that short-term HFD feeding leads to acute proinflammatory signals in the hypothalamus without significant leukocyte activation in other tissues that may be largely reversible (65). In the setting of chronic obesity, while multiple tissue-specific triggers may be activated, there is evidence to suggest that circulating inflammatory mediators are induced by obesity and generate broad effects on tissue inflammation.

The important role of innate immune responses to bacterial products such as endotoxins and LPS has advanced the hypothesis of metabolic endotoxemia as both acute and chronic stimuli for metabolic tissues $(93,98)$. This concept is supported by the observations that circulating endotoxin levels are higher in diabetic adults compared with non-diabetic adults (99). Obese mothers have two-fold higher endotoxin levels associated with elevated ATMs and inflammatory cytokine secretion from adipose tissue (100). In obese children, inflammatory biomarkers associated with cardiovascular risk, such as plasminogen activator inhibitor 1 and TNF- $\alpha$, correlate with serum endotoxin levels (101). Animal models suggest that HFD feeding compromises gut barrier function that contributes directly to endotoxemia and that gutdelivered antiinflammatory agents may limit systemic LPS (93). Innate pattern recognition receptors such as toll-like receptors are present on multiple metabolic cells, and LPS has been shown to 
negatively influence substrate metabolism, storage, and absorption in these tissues $(93,102-104)$. In adipose tissue, experimental endotoxin infusion in humans rapidly induces chemokine and $\mathrm{T}$ cell activation genes and therefore may potentiate leukocyte crosstalk and amplify initial inflammatory responses (105). This concept still remains somewhat controversial, as limitations in endotoxin measurement methods prevent the wide use of endotoxin as a true biomarker for metainflammation (106). However, the observation that treatment of obese adolescents leads to both improvements in insulin resistance and decreased endotoxin levels suggests that early interventions may limit metabolic endotoxemia (107).

Another initiating event in metainflammation is the direct influence of the obesogenic environment on the generation of leukocytes and the potentiation of their responses to tissue stress and damage (108). The observation of increased circulating neutrophils and monocytes in obese children suggests that obesity fundamentally alters leukocyte production and/or turnover, and this has been supported in preclinical studies. Bone marrow-derived hematopoietic stem cells (HSCs) are the primary source of mature leukocytes (109) and have the capacity to alter their cellular output in response to environmental cues (110). For example, in sepsis, endotoxin can "push" HSCs to increase myeloid cell production and potentiate innate immune responses (111).

A similar stimulation and expansion of HSCs in obesity may be a mechanism by which activated myeloid responses can be propagated to different metabolic tissues $(112,113)$. Nutrients can directly stimulate myelopoiesis from HSCs. For example, hyperglycemia can induce the calcium-binding proteins S100A8 and S100A9 and stimulate monocyte production from myeloid progenitor cells in models of type 1 diabetes (114). Fish oil-rich diets promote hematopoiesis through the activation of MMP12 in the bone marrow (115). TLR4 activation induces HSC expansion and myelopoiesis and may be another mechanism by which metabolic endotoxemia imparts its effects on metainflammation (116-118). ATMs can generate inflammasome-dependent signals that activate bone marrow granulocyte and macrophage progenitors via IL-1 $\beta$ (119). In addition, obesity may also influence HSC function indirectly by modifying the bone marrow niche populated with specialized adipocytes (120). The obese gut microbiome has been implicated in stimulating myelopoiesis via modification of the bone marrow niche (121).

As in metabolic tissues, epigenetic regulation of the immune system plays crucial roles in leukocyte production and in altered immune responses after severe illness such as sepsis (122). Such epigenetic programming in leukocytes by obesity may explain why the risk for diabetes is sustained in formerly obese adults and why persistent adipose tissue inflammation is seen after weight loss $(123,124)$. HSCs from obese mice have the enhanced capacity to generate proinflammatory $\mathrm{CD} 11 \mathrm{c}^{+}$ATMs compared with lean HSCs, even after serial bone marrow transplantation (112), suggesting that HSC-autonomous mechanisms contribute to metainflammation. Obesity can alter methylation patterns in circulating lymphocytes (125) and modify histone methylation of genes regulating macrophage production in the bone marrow such as IL12 (126). Differential DNA methylation is seen in adipose tissue from individuals with type 2 diabetes, and target genes include those involved in inflammation and carbohydrate and lipid metabolism (127). Similar mechanisms may contribute to the impaired fetal hematopoiesis that is observed with maternal obesity (128). There is a paucity of information about the point at which epigenetic alterations may occur in obese children; however, it is reasonable to postulate that early nutritional stress associated with rapid weight gain may trigger long-term alterations in immune function.

The quantitative and qualitative alterations in HSCs and leukocytes are potential priming events that shift a healthy adaptive inflammatory response to one that is deleterious to homeostasis. This may occur either by increasing the amplitude or the duration of the inflammatory response to adipocyte stress and death. VAT is a site where these competing forces are held in a delicate balance to maintain the robust ability to store and release nutrients on demand. Under the stress of excess nutrient availability, hypertrophic adipocytes are subjected to stress, leading to cellular degeneration that activates the inflammasome and initiates proinflammatory programmed cell death (pyroptosis) (129). Hallmarks of adipose tissue that has lost this flexibility include fibrosis and inflammatory clusters of leukocytes in CLSs $(130,131)$, both of which have been observed in obese children and adults. Subsequent production of chemoattractants such as CCL2 and lipolytic signals triggers the recruitment of monocytes from the circulation to form the CLS that engulfs the dying adipocytes and potentiates new adipocyte formation $(132,133)$. Mouse models in which macrophage recruitment or activation are attenuated show a phenotype of permissive adipocyte hypertrophy, reduced adipocyte death, and preservation of metabolic health, suggesting that macrophages may accelerate adipocyte death and not merely passively respond to it (21). VAT CLS formation is seen in obese children, suggesting that these events are initiated in early life and not only in obese adults $(134,135)$. CD206 ${ }^{+}$ATMs are prominent in adipose tissue from children and are positively associated with adipocyte size (136). However, the existing VAT gene expression profiling data from obese and non-obese children demonstrates a relative paucity of differentially expressed inflammatory genes (137). This contrasts with what is seen in obese adult VAT, which is enriched for inflammatory gene pathways $(138,139)$. These findings suggest that the nature of the metainflammatory response in adipose tissue may be fundamentally different in children despite the similar formation of CLSs. It may be that the duration of obesity influences the magnitude of the inflammatory response or that children retain more of the homeostatic pathways meant to dampen inflammation despite the same stimulus of adipocyte stress.

\section{Conclusion}

Obesity has lifelong effects on metabolism and immune system activation that may have multiple starting points from the prenatal period through adolescence. The existing data suggest that whenever it is initiated in children, many features of metainflammation persist through adulthood. Weight reduction in children can normalize inflammatory markers, which gives us hope that metainflammation can be modified after it has begun (140). However, there remain many gaps in our understanding of how early life events (e.g., rapid post-natal growth) affect lifelong risk for metabolic disease and what interventions may be tenable in this 
window of development. Advancing this line of inquiry will require efforts such as the NIH Environmental influences on Child Health Outcomes (ECHO) Program (141), which has replaced the National Children's Study and will leverage extant cohorts to understand how environmental exposures influence pediatric outcomes such as obesity. Environmental exposure is broadly defined to encompass the biological, chemical, behavioral, social, physical, and built environments that have undergone major changes in the past several decades in concert with the increase in obesity. This program provides an opportunity for scientists and physicians to engage with a broader research community to solve this wide-ranging problem.

Reframing metainflammation and metabolic disease as a pediatric morbidity also provides opportunities for discovery in basic science by framing inflammation in a developmental context. All pediatric chronic diseases require a developmental biology framework to deliver optimal care and to understand their pathogenesis. It is clear that a similar framework is required to understand how metainflammation begins (Figure 1). Investigations focused on these early inciting events have the potential to identify preventative therapeutics for metainflammation that are currently lacking. A careful intersection between basic and clinical research is needed to fully identify the mechanisms involved in the initiation of metainflammation during childhood. This is particularly important given the known limitations of mice for the study of immunology, as current husbandry practices generate mice with persistent immature immune systems with features that more closely match newborn humans than adult humans (142). This developmental perspective is not unique to children and is also required to evaluate adult metabolic disease, given the diversity of settings in which metabolic health is compromised throughout an individual's lifespan.

\section{Acknowledgments}

KS is supported by grants from the NIH/NIDDK (DK101755) and the SKS/Edith Briskin Taubman Emerging Scholars funds. CNL is supported by grants from the NIH/NIDDK (DK090262, HD086696) and the American Diabetes Association (7-12-CD-08).

Address correspondence to: Carey Lumeng, University of Michigan Medical School, Department of Pediatrics and Communicable Diseases, 2057 BSRB, 109 Zina Pitcher Place, Ann Arbor, Michigan 48109-2200, USA. Phone: 734.615.6242; E-mail: clumeng @umich.edu.
1. Ogden CL, Carroll MD, Kit BK, Flegal KM. Prevalence of childhood and adult obesity in the United States, 2011-2012. JAMA. 2014;311(8):806-814.

2. Hammond RA, Levine R. The economic impact of obesity in the United States. Diabetes Metab Syndr Obes. 2010;3:285-295.

3. Skinner AC, Perrin EM, Skelton JA. Prevalence of obesity and severe obesity in US children, 19992014. Obesity (Silver Spring). 2016;24(5):1116-1123.

4. Miller JM, Kaylor MB, Johannsson M, Bay C, Churilla JR. Prevalence of metabolic syndrome and individual criterion in US adolescents: 2001-2010 National Health and Nutrition Examination Survey. Metab Syndr Relat Disord. 2014;12(10):527-532.

5. Agirbasli M, Tanrikulu AM, Berenson GS. Metabolic syndrome: bridging the gap from childhood to adulthood. Cardiovasc Ther. 2016;34(1):30-36.

6. Kotas ME, Medzhitov R. Homeostasis, inflammation, and disease susceptibility. Cell. 2015;160(5):816-827.

7. Medzhitov R. Origin and physiological roles of inflammation. Nature. 2008;454(7203):428-435.

8. Spitzer JA, Kovach AG, Rosell S, Sandor P, Spitzer JJ, Storck R. Influence of endotoxin on adipose tissue metabolism. Adv Exp Med Biol. 1972;33(0):337-344.

9. Hotamisligil GS, Shargill NS, Spiegelman BM. Adipose expression of tumor necrosis factoralpha: direct role in obesity-linked insulin resistance. Science. 1993;259(5091):87-91.

10. Mathis D. Immunological goings-on in visceral adipose tissue. Cell Metab. 2013;17(6):851-859.

11. Vandanmagsar B, et al. The NLRP3 inflammasome instigates obesity-induced inflammation and insulin resistance. Nat Med. 2011;17(2):179-188.

12. Wen $\mathrm{H}$, et al. Fatty acid-induced NLRP3-ASC inflammasome activation interferes with insulin signaling. Nat Immunol. 2011;12(5):408-415.

13. Xu X, Grijalva A, Skowronski A, van Eijk M, Serlie
MJ, Ferrante AW. Obesity activates a program of lysosomal-dependent lipid metabolism in adipose tissue macrophages independently of classic activation. Cell Metab. 2013;18(6):816-830.

14. Solinas G, et al. JNK1 in hematopoietically derived cells contributes to diet-induced inflammation and insulin resistance without affecting obesity. Cell Metab. 2007;6(5):386-397.

15. Wernstedt Asterholm I, et al. Adipocyte inflammation is essential for healthy adipose tissue expansion and remodeling. Cell Metab. 2014;20(1):103-118.

16. Ji Y, Sun S, Xia S, Yang L, Li X, Qi L. Short term high fat diet challenge promotes alternative macrophage polarization in adipose tissue via natural killer T cells and interleukin-4. J Biol Chem. 2012;287(29):24378-24386.

17. Lee YS, et al. Inflammation is necessary for longterm but not short-term high-fat diet-induced insulin resistance. Diabetes. 2011;60(10):2474-2483.

18. Thaler JP, et al. Obesity is associated with hypothalamic injury in rodents and humans. J Clin Invest. 2012;122(1):153-162.

19. Molofsky AB, et al. Innate lymphoid type 2 cells sustain visceral adipose tissue eosinophils and alternatively activated macrophages. JExp Med. 2013;210(3):535-549.

20. Amano SU, et al. Local proliferation of macrophages contributes to obesity-associated adipose tissue inflammation. Cell Metab. 2014;19(1):162-171.

21. Weisberg SP, et al. CCR2 modulates inflammatory and metabolic effects of high-fat feeding. J Clin Invest. 2006;116(1):115-124.

22. Talukdar S, et al. Neutrophils mediate insulin resistance in mice fed a high-fat diet through secreted elastase. Nat Med. 2012;18(9):1407-1412.

23. Mansuy-Aubert V, et al. Imbalance between neutrophil elastase and its inhibitor $\alpha 1$-antitrypsin in obesity alters insulin sensitivity, inflammation, and energy expenditure. Cell Metab. 2013;17(4):534-548.
24. Feuerer M, et al. Lean, but not obese, fat is enriched for a unique population of regulatory $\mathrm{T}$ cells that affect metabolic parameters. Nat Med. 2009;15(8):930-939.

25. Lumeng CN, Bodzin JL, Saltiel AR. Obesity induces a phenotypic switch in adipose tissue macrophage polarization. J Clin Invest. 2007;117(1):175-184.

26. Iyengar NM, et al. Systemic correlates of white adipose tissue inflammation in early-stage breast cancer. Clin Cancer Res. 2016;22(9):2283-2289.

27. Kratz M, et al. Metabolic dysfunction drives a mechanistically distinct proinflammatory phenotype in adipose tissue macrophages. Cell Metab. 2014;20(4):614-625.

28. Morris DL, et al. Adipose tissue macrophages function as antigen-presenting cells and regulate adipose tissue $\mathrm{CD} 4^{+} \mathrm{T}$ cells in mice. Diabetes. 2013;62(8):2762-2772.

29. Moraes-Vieira PM, Yore MM, Dwyer PM, Syed I, Aryal P, Kahn BB. RBP4 activates antigenpresenting cells, leading to adipose tissue inflammation and systemic insulin resistance. Cell Metab. 2014;19(3):512-526.

30. Winer S, et al. Normalization of obesity-associated insulin resistance through immunotherapy: $\mathrm{CD}^{+}{ }^{+} \mathrm{T}$ cells control glucose homeostasis. $\mathrm{Nat}$ Med. 2009;15(8):921-929.

31. Tomar AS, Tallapragada DS, Nongmaithem SS, Shrestha S, Yajnik CS, Chandak GR. Intrauterine Programming of Diabetes and Adiposity. Curr Obes Rep. 2015;4(4):418-428.

32. Alfaradhi MZ, et al. Maternal obesity in pregnancy developmentally programs adipose tissue inflammation in young, lean male mice offspring. Endocrinology. 2016;157(11):4246-4256.

33. Lecoutre S, Breton C. The cellularity of offspring's adipose tissue is programmed by maternal nutritional manipulations. Adipocyte. 2014;3(4):256-262.

34. Murabayashi N, et al. Maternal high-fat diets 
cause insulin resistance through inflammatory changes in fetal adipose tissue. Eur JObstet Gynecol Reprod Biol. 2013;169(1):39-44.

35. Ornellas F, Souza-Mello V, Mandarim-de-Lacerda CA, Aguila MB. Combined parental obesity augments single-parent obesity effects on hypothalamus inflammation, leptin signaling (JAK/ STAT), hyperphagia, and obesity in the adult mice offspring. Physiol Behav. 2016;153:47-55.

36. Ding Y, et al. DNA hypomethylation of inflammation-associated genes in adipose tissue of female mice after multigenerational high fat diet feeding. Int JObes (Lond). 2014;38(2):198-204.

37. Pantham P, Aye IL, Powell TL. Inflammation in maternal obesity and gestational diabetes mellitus. Placenta. 2015;36(7):709-715.

38. Challier JC, et al. Obesity in pregnancy stimulates macrophage accumulation and inflammation in the placenta. Placenta. 2008;29(3):274-281.

39. Roberts KA, et al. Placental structure and inflammation in pregnancies associated with obesity. Placenta. 2011;32(3):247-254.

40. van der Burg JW, et al. Is maternal obesity associated with sustained inflammation in extremely low gestational age newborns? Early Hum Dev. 2013;89(12):949-955.

41. Leibowitz KL, et al. Maternal obesity associated with inflammation in their children. World J Pediatr. 2012;8(1):76-79.

42. Barker DJ. Fetal origins of coronary heart disease. BMJ. 1995;311(6998):171-174.

43. Barker DJ, Hales CN, Fall CH, Osmond C, Phipps K, Clark PM. Type 2 (non-insulin-dependent) diabetes mellitus, hypertension and hyperlipidaemia (syndrome X): relation to reduced fetal growth. Diabetologia. 1993;36(1):62-67.

44. Yajnik CS. Transmission of obesity-adiposity and related disorders from the mother to the baby. Ann Nutr Metab. 2014;64(suppl 1):8-17.

45. Magee TR, Han G, Cherian B, Khorram O, Ross MG, Desai M. Down-regulation of transcription factor peroxisome proliferator-activated receptor in programmed hepatic lipid dysregulation and inflammation in intrauterine growth-restricted offspring. Am JObstet Gynecol. 2008;199(3):271.e1-271.e5.

46. Riddle ES, et al. Intrauterine growth restriction increases TNF $\alpha$ and activates the unfolded protein response in male rat pups. JObes. 2014;2014:829862.

47. Boutsikou T, et al. Circulating levels of inflammatory markers in intrauterine growth restriction. Mediators Inflamm. 2010;2010:790605.

48. Sutton GM, Centanni AV, Butler AA. Protein malnutrition during pregnancy in C57BL/6J mice results in offspring with altered circadian physiology before obesity. Endocrinology. 2010;151(4):1570-1580.

49. Chen W, Srinivasan SR, Berenson GS. Influence of birth weight on white blood cell count in biracial (black-white) children, adolescents, and young adults: the Bogalusa Heart Study. Am J Epidemiol. 2009;169(2):214-218.

50. Bhuiyan AR, Srinivasan SR, Chen W, Azevedo MJ, Berenson GS. Influence of low birth weight on C-reactive protein in asymptomatic younger adults: the bogalusa heart study. BMC Res Notes. 2011;4:71.
51. Stansfield BK, Fain ME, Bhatia J, Gutin B, Nguyen JT, Pollock NK. Nonlinear relationship between birth weight and visceral fat in adolescents. JPediatr. 2016;174:185-192.

52. Zhang Z, Kris-Etherton PM, Hartman TJ. Birth weight and risk factors for cardiovascular disease and type 2 diabetes in US children and adolescents: 10 year results from NHANES. Matern Child Health J. 2014;18(6):1423-1432.

53. Gensollen T, Iyer SS, Kasper DL, Blumberg RS. How colonization by microbiota in early life shapes the immune system. Science. 2016;352(6285):539-544.

54. Dowling DJ, Levy O. Ontogeny of early life immunity. Trends Immunol. 2014;35(7):299-310.

55. Kolodin D, et al. Antigen- and cytokine-driven accumulation of regulatory $\mathrm{T}$ cells in visceral adipose tissue of lean mice. Cell Metab. 2015;21(4):543-557.

56. Ashino NG, et al. Maternal high-fat feeding through pregnancy and lactation predisposes mouse offspring to molecular insulin resistance and fatty liver. J Nutr Biochem. 2012;23(4):341-348.

57. Rother E, et al. Hypothalamic JNK1 and IKK $\beta$ activation and impaired early postnatal glucose metabolism after maternal perinatal high-fat feeding. Endocrinology. 2012;153(2):770-781.

58. Plagemann A, Heidrich I, Götz F, Rohde W, Dörner G. Obesity and enhanced diabetes and cardiovascular risk in adult rats due to early postnatal overfeeding. Exp Clin Endocrinol. 1992;99(3):154-158.

59. Conceição EP, et al. Oxidative stress programming in a rat model of postnatal early overnutrition - role of insulin resistance. J Nutr Biochem. 2013;24(1):81-87.

60. Glavas MM, et al. Early overnutrition results in early-onset arcuate leptin resistance and increased sensitivity to high-fat diet. Endocrinology. 2010;151(4):1598-1610.

61. Ziko I, et al. Neonatal overfeeding alters hypothalamic microglial profiles and central responses to immune challenge long-term. Brain Behav Immun. 2014;41:32-43.

62. Tapia-González S, et al. Activation of microglia in specific hypothalamic nuclei and the cerebellum of adult rats exposed to neonatal overnutrition. J Neuroendocrinol. 2011;23(4):365-370.

63. Cai G, et al. Overfeeding during a critical postnatal period exacerbates hypothalamicpituitary-adrenal axis responses to immune challenge: a role for adrenal melanocortin 2 receptors. Sci Rep. 2016;6:21097.

64. Cai G, et al. Neonatal overfeeding attenuates acute central pro-inflammatory effects of shortterm high fat diet. Front Neurosci. 2014;8:446.

65 . Berkseth KE, et al. Hypothalamic gliosis associated with high-fat diet feeding is reversible in mice: a combined immunohistochemical and magnetic resonance imaging study. Endocrinology. 2014;155(8):2858-2867.

66. Liu Z, et al. Neonatal overnutrition in mice exacerbates high-fat diet-induced metabolic perturbations. JEndocrinol. 2013;219(2):131-143.

67. Kayser BD, Goran MI, Bouret SG. Perinatal overnutrition exacerbates adipose tissue inflammation caused by high-fat feeding in C57BL/6J mice. PLoS One. 2015;10(3):e0121954.

68. Ye Z, et al. Obesity induced by neonatal overfeeding worsens airway hyperresponsiveness and inflammation. PLoS One. 2012;7(10):e47013.

69. Clarke MA, Stefanidis A, Spencer SJ. Postnatal overfeeding leads to obesity and exacerbated febrile responses to lipopolysaccharide throughout life. JNeuroendocrinol. 2012;24(3):511-524.

70. Beardsall K, et al. Heritability of childhood weight gain from birth and risk markers for adult metabolic disease in prepubertal twins. J Clin Endocrinol Metab. 2009;94(10):3708-3713.

71. Ong KK, et al. Infancy weight gain predicts childhood body fat and age at menarche in girls. JClin Endocrinol Metab. 2009;94(5):1527-1532.

72. Demerath EW, et al. Rapid postnatal weight gain and visceral adiposity in adulthood: the Fels Longitudinal Study. Obesity (Silver Spring). 2009;17(11):2060-2066.

73. Kerkhof GF, Leunissen RW, Hokken-Koelega AC. Early origins of the metabolic syndrome: role of small size at birth, early postnatal weight gain, and adult IGF-I. J Clin Endocrinol Metab. 2012;97(8):2637-2643.

74. Elks CE, et al. Associations between genetic obesity susceptibility and early postnatal fat and lean mass: an individual participant meta-analysis. JAMA Pediatr. 2014;168(12):1122-1130.

75. Skilton MR, et al. Weight gain in infancy and vascular risk factors in later childhood. Pediatrics. 2013;131(6):e1821-e1828.

76. Magnus MC, et al. Infant growth and risk of childhood-onset type 1 diabetes in children from 2 Scandinavian birth cohorts. JAMA Pediatr. 2015;169(12):e153759.

77. den Dekker HT, et al. Early growth characteristics and the risk of reduced lung function and asthma: a meta-analysis of 25,000 children. J Allergy Clin Immunol. 2016;137(4):1026-1035.

78. Deshmukh-Taskar P, Nicklas TA, Morales M, Yang SJ, Zakeri I, Berenson GS. Tracking of overweight status from childhood to young adulthood: the Bogalusa Heart Study. Eur J Clin Nutr. 2006;60(1):48-57.

79. Attard SM, Herring AH, Howard AG, Gordon-Larsen P. Longitudinal trajectories of BMI and cardiovascular disease risk: the nationa longitudinal study of adolescent health. Obesity (Silver Spring). 2013;21(11):2180-2188.

80. Power C, Thomas C. Changes in BMI, duration of overweight and obesity, and glucose metabolism: 45 years of follow-up of a birth cohort. Diabetes Care. 2011;34(9):1986-1991.

81. Ferrari M, et al. Inflammation profile in overweight/obese adolescents in Europe: an analysis in relation to iron status. Eur J Clin Nutr. 2015;69(2):247-255.

82. Skinner AC, Steiner MJ, Henderson FW, Perrin EM. Multiple markers of inflammation and weight status: cross-sectional analyses throughout childhood. Pediatrics. 2010;125(4):e801-e809.

83. Chen W, Srinivasan SR, Xu J, Berenson GS. Blackwhite divergence in the relation of white blood cell count to metabolic syndrome in preadolescents, adolescents, and young adults: the Bogalusa Heart Study. Diabetes Care. 2010;33(11):2474-2476.

84. Nazmi A, Gonzalez DC, Oliveira IO, Horta BL, Gigante DP, Victora CG. Life course weight gain 
and C-reactive protein levels in young adults: findings from a Brazilian birth cohort. Am J Hum Biol. 2009;21(2):192-199.

85. Gong L, et al. Weight loss, inflammatory markers, and improvements of iron status in overweight and obese children. JPediatr. 2014;164(4):795-800.e2.

86. Singer K, Eng DS, Lumeng CN, Gebremariam A, $\mathrm{M}$ Lee J. The relationship between body fat mass percentiles and inflammation in children. Obesity (Silver Spring). 2014;22(5):1332-1336.

87. Michalsky MP, et al. Cardiovascular Risk Factors in Severely Obese Adolescents: The Teen Longitudinal Assessment of Bariatric Surgery (Teen-LABS) Study. JAMA Pediatr. 2015;169(5):438-444.

88. Weiss R, et al. Obesity and the metabolic syndrome in children and adolescents. $N$ Engl J Med. 2004;350(23):2362-2374

89. Xanthakos SA, et al. High prevalence of nonalcoholic fatty liver disease in adolescents undergoing bariatric surgery. Gastroenterology. 2015;149(3):623-34.e8.

90. Walker RW, et al. Macrophages and fibrosis in adipose tissue are linked to liver damage and metabolic risk in obese children. Obesity (Silver Spring). 2014;22(6):1512-1519.

91. Kursawe R, et al. A role of the inflammasome in the low storage capacity of the abdominal subcutaneous adipose tissue in obese adolescents. Diabetes. 2016;65(3):610-618.

92. Landgraf K, et al. Evidence of early alterations in adipose tissue biology and function and its association with obesity-related inflammation and insulin resistance in children. Diabetes. 2015;64(4):1249-1261.

93. Luck H, et al. Regulation of obesity-related insulin resistance with gut anti-inflammatory agents. Cell Metab. 2015;21(4):527-542.

94. Stefanovic-Racic M, et al. Dendritic cells promote macrophage infiltration and comprise a substantial proportion of obesity-associated increases in CD11c+ cells in adipose tissue and liver. Diabetes. 2012;61(9):2330-2339.

95. Eguchi K, et al. Saturated fatty acid and TLR signaling link $\beta$ cell dysfunction and islet inflammation. Cell Metab. 2012;15(4):518-533.

96. Fink LN, et al. Pro-inflammatory macrophages increase in skeletal muscle of high fat-fed mice and correlate with metabolic risk markers in humans. Obesity (Silver Spring). 2014;22(3):747-757.

97. Buckman LB, et al. Obesity induced by a highfat diet is associated with increased immune cell entry into the central nervous system. Brain Behav Immun. 2014;35:33-42.

98. Cani PD, et al. Metabolic endotoxemia initiates obesity and insulin resistance. Diabetes. 2007;56(7):1761-1772.

99. Pussinen PJ, Havulinna AS, Lehto M, Sundvall J, Salomaa V. Endotoxemia is associated with an increased risk of incident diabetes. Diabetes Care. 2011;34(2):392-397.

100. Basu S, et al. Pregravid obesity associates with increased maternal endotoxemia and metabolic inflammation. Obesity (Silver Spring). 2011;19(3):476-482.

101.Varma MC, et al. Metabolic endotoxaemia in childhood obesity. BMC Obes. 2015;3:3.

102. Pohl J, Woodside B, Luheshi GN. Changes in hypothalamically mediated acute-phase inflammatory responses to lipopolysaccharide in diet-induced obese rats. Endocrinology. 2009;150(11):4901-4910

103. Creely SJ, et al. Lipopolysaccharide activates an innate immune system response in human adipose tissue in obesity and type 2 diabetes. Am J Physiol Endocrinol Metab. 2007;292(3):E740-E747.

104. Frisard MI, et al. Toll-like receptor 4 modulates skeletal muscle substrate metabolism. Am J Physiol Endocrinol Metab. 2010; 298(5):E988-E998.

105. Shah R, et al. Gene profiling of human adipose tissue during evoked inflammation in vivo. Diabetes. 2009;58(10):2211-2219.

106. Boutagy NE, McMillan RP, Frisard MI, Hulver MW. Metabolic endotoxemia with obesity: Is it real and is it relevant? Biochimie. 2016;124:11-20.

107. Lira FS, et al. Long-term interdisciplinary therapy reduces endotoxin level and insulin resistance in obese adolescents. Nutr J. 2012;11:74.

108. Schipper HS, et al. Systemic inflammation in childhood obesity: circulating inflammatory mediators and activated CD $14^{++}$monocytes. Diabetologia. 2012;55(10):2800-2810.

109. Granick JL, Simon SI, Borjesson DL. Hematopoietic stem and progenitor cells as effectors in innate immunity. Bone Marrow Res. 2012;2012:165107.

110. King KY, Goodell MA. Inflammatory modulation of HSCs: viewing the HSC as a foundation for the immune response. Nat Rev Immunol. 2011;11(10):685-692

111. Esplin BL, et al. Chronic exposure to a TLR ligand injures hematopoietic stem cells. Jimmunol. 2011;186(9):5367-5375

112. Singer K, et al. Diet-induced obesity promotes myelopoiesis in hematopoietic stem cells. $\mathrm{Mol}$ Metab. 2014;3(6):664-675.

113. Bellows CF, Zhang Y, Simmons PJ, Khalsa AS, Kolonin MG. Influence of BMI on level of circulating progenitor cells. Obesity (Silver Spring). 2011;19(8):1722-1726.

114. Nagareddy PR, et al. Hyperglycemia promotes myelopoiesis and impairs the resolution of ath erosclerosis. Cell Metab. 2013;17(5):695-708.

115. Xia S, et al. Fish oil-rich diet promotes hematopoiesis and alters hematopoietic niche. Endocrinology. 2015;156(8):2821-2830.

116. Shi H, Kokoeva MV, Inouye K, Tzameli I, Yin H, Flier JS. TLR4 links innate immunity and fatty acid-induced insulin resistance. JClin Invest. 2006;116(11):3015-3025

117. Megías J, Yáñez A, Moriano S, O’Connor JE, Gozalbo D, Gil ML. Direct Toll-like receptormediated stimulation of hematopoietic stem and progenitor cells occurs in vivo and promotes differentiation toward macrophages. Stem Cells. 2012;30(7):1486-1495.

118. Nagai Y, et al. Toll-like receptors on hematopoietic progenitor cells stimulate innate immune system replenishment. Immunity. 2006;24(6):801-812.

119. Nagareddy PR, et al. Adipose tissue macrophages promote myelopoiesis and monocytosis in obesi- ty. Cell Metab. 2014;19(5):821-835.

120. Cawthorn WP, et al. Bone marrow adipose tissue is an endocrine organ that contributes to increased circulating adiponectin during caloric restriction. Cell Metab. 2014;20(2):368-375.

121. Luo Y, et al. Microbiota from obese mice regulate hematopoietic stem cell differentiation by altering the bone niche. Cell Metab. 2015;22(5):886-894.

122. Carson WF, Cavassani KA, Dou Y, Kunkel SL. Epigenetic regulation of immune cell functions during post-septic immunosuppression. Epigenetics. 2011;6(3):273-283.

123. Kratz M, et al. Improvements in glycemic contro after gastric bypass occur despite persistent adipose tissue inflammation. Obesity (Silver Spring). 2016;24(7):1438-1445.

124. Cancello R, et al. Permanence of molecular features of obesity in subcutaneous adipose tissue of ex-obese subjects. Int J Obes (Lond) 2013;37(6):867-873.

125. Jacobsen MJ, et al. Altered methylation profile of lymphocytes is concordant with perturbation of lipids metabolism and inflammatory response in obesity. J Diabetes Res. 2016;2016:8539057.

126. Gallagher KA, et al. Epigenetic changes in bone marrow progenitor cells influence the inflammatory phenotype and alter wound healing in type 2 diabetes. Diabetes. 2015;64(4):1420-1430.

127. Nilsson E, et al. Altered DNA methylation and differential expression of genes influencing metabolism and inflammation in adipose tissue from subjects with type 2 diabetes. Diabetes. 2014;63(9):2962-2976.

128. Kamimae-Lanning AN, et al. Maternal high-fat diet and obesity compromise fetal hematopoiesis. Mol Metab. 2015;4(1):25-38.

129. Giordano A, et al. Obese adipocytes show ultrastructural features of stressed cells and die of pyroptosis. J Lipid Res. 2013;54(9):2423-2436.

130. Sun K, Tordjman J, Clement K, Scherer PE. Fibrosis and adipose tissue dysfunction. Cell Metab. 2013;18(4):470-477.

131. Murano I, et al. Dead adipocytes, detected as crown-like structures (CLS), are prevalent in visceral fat depots of genetically obese mice. J Lipid Res. 2008;49(7):1562-1568.

132. Kosteli A, et al. Weight loss and lipolysis promote a dynamic immune response in murine adipose tissue. J Clin Invest. 2010;120(10):3466-3479.

133. Lee YH, Petkova AP, Granneman JG. Identification of an adipogenic niche for adipose tissue remodeling and restoration. Cell Metab. 2013;18(3):355-367.

134. Tam CS, et al. An early inflammatory gene profile in visceral adipose tissue in children. Int J Pediatr Obes. 2011;6(2-2):e360-e363.

135. Sbarbati A, et al. Obesity and inflammation: evidence for an elementary lesion. Pediatrics. 2006;117(1):220-223.

136. Tam CS, Tordjman J, Divoux A, Baur LA, Clément K. Adipose tissue remodeling in children: the link between collagen deposition and age-related adipocyte growth. J Clin Endocrinol Metab. 2012;97(4):1320-1327.

137. Aguilera CM, Gomez-Llorente C, Tofe I, Gil-Campos M, Cañete R, Gil Á. Genome- 
wide expression in visceral adipose tissue from obese prepubertal children. Int JMol Sci. 2015;16(4):7723-7737.

138. Hardy OT, et al. Body mass index-independent inflammation in omental adipose tissue associated with insulin resistance in morbid obesity. Surg Obes Relat Dis. 2011;7(1):60-67.
139. Chen Y, et al. Variations in DNA elucidate molecular networks that cause disease. Nature. 2008;452(7186):429-435.

140. Tzoulaki I, et al. Size at birth, weight gain over the life course, and low-grade inflammation in young adulthood: northern Finland 1966 Birth Cohort study. Eur Heart J. 2008;29(8):1049-1056.
141. NIH. Environmental influences on Child Health Outcomes (ECHO) Program. NIH Web site. https://www.nih.gov/echo. Accessed October 24, 2016.

142. Beura LK, et al. Normalizing the environment recapitulates adult human immune traits in laboratory mice. Nature. 2016;532(7600):512-516. 